\title{
De ciel en ciel: lecture de deux romans de Béroalde de Verville
}

\section{Daniela Mauri}

\section{(2) OpenEdition}

1 Journals

Édition électronique

URL : http://journals.openedition.org/studifrancesi/16064

DOI : 10.4000/studifrancesi. 16064

ISSN : 2421-5856

Éditeur

Rosenberg \& Sellier

\section{Édition imprimée}

Date de publication : 1 juillet 2019

Pagination : 5-18

ISSN : 0039-2944

\section{Référence électronique}

Daniela Mauri, « De ciel en ciel: lecture de deux romans de Béroalde de Verville », Studi Francesi [En ligne], 187 (LXIII | I) | 2019, mis en ligne le 01 avril 2020, consulté le 25 janvier 2021. URL : http:// journals.openedition.org/studifrancesi/16064; DOI : https://doi.org/10.4000/studifrancesi.16064

\section{(c) (†) $\odot$}

Studi Francesi è distribuita con Licenza Creative Commons Attribuzione - Non commerciale - Non opere derivate 4.0 Internazionale. 


\title{
De ciel en ciel: lecture de deux romans de Béroalde de Verville
}

\begin{abstract}
In this paper I will deal with the sky, seen as a place of divinity and as a celestial space in two novels by the baroque writer Béroalde de Verville: Les Amours d' Æsionne (1597) and La Pucelle d'Orléans (1599). These novels have in common the themes of war and love. But they are different as regards the evocation of the celestial space, linked above all to the action of Fortune and Destiny in the first work, while, in the second, it is closely connected to the religious theme, perhaps because of its protagonist, which is a heroine in the Christian tradition. She is, however, transformed by Béroalde into a character in communication with "intelligences supérieures" or "puissances séparées" (that are not better identified), becoming a sort of syncretic figure. These entities are also present in Æsionne. One of the main characters in this novel is in fact the Flambor hermit who brings together in himself the power of a magician, of a quasi-deity in possession of a superior knowledge obtained through contact with the "puissances célestes".
\end{abstract}

Béroalde de Verville ${ }^{1}$, auteur très prolifique de l'époque baroque, exprime un intérêt très évident pour le ciel et l'espace céleste, qui constituent une présence constante dès le début de sa production tant d'un point de vue religieux que d'un point de vue plus spécifiquement narratif. Dans cette étude nous analyserons, selon cet aspect particulier, deux de ses romans principaux: Les Amours d'Æsionne et La

(1) Béroalde de Verville, dont le vrai nom était François Brouard ou Brouart, était fils de Matthieu Béroalde, qui avait été le maître d'Agrippa d'Aubigné et de Pierre de L'Estoile. Notre auteur hérita de son père l'amour pour l'étude et la connaissance. Il fut un vrai “curieux" et il s'occupa de différentes matières: les mathématiques, l'architecture, la mécanique, la médecine, l’optique. Les données relatives à sa biographie ne sont pas très abondantes. Appartenant à une famille protestante, il dut passer au catholicisme, puisque, en 1593, il avait été nommé chanoine de Saint-Gatien de Tours. Il est toutefois probable qu'il s'était encore tourné vers le protestantisme peu avant sa mort. La bibliographie critique sur cet auteur est désormais très riche, surtout en ce qui concerne son ouvrage principal, Le Moyen de parvenir. Nous citons tout d'abord les références bibliographiques essentielles: V.-L. SAULnIER, Étude sur Béroalde de Verville, in «Bibliothèque d'Humanisme et Renaissance», T.V., 1944, pp. 209-326 (cette étude peut être considérée comme le travail 'fondateur' de la critique concernant cet écrivain). V. aussi le répertoires bibliographiques de M. Giordano, J. Pallister, Le Moyen de parvenir: Bibliographic Notes, «Papers on French Seventeenth Century Literature», 1981, et M. GiordanO, I. Zinguer, Bibliography 1981-1991, in Studies on Béroalde de Verville, «Papers on French Seventeenth Century Literature», 1992, pp. 139-147. V. aussi l'étude fondamentale de N. Kenny, The Palace of Secrets - Béroalde de Verville and Renaissance Conception of Knowledge, Oxford, Clarendon Press, 1991, et les actes du premier colloque international consacré à notre auteur (Paris-Sorbonne, 9 mars 1995): Béroalde de Verville (1556-1626), Paris, Presses de l'École Normale Supérieure, 1996, «Cahiers V.-L. Saulnier» 13. Ce volume contient aussi une bibliographie sur Béroalde par A.Tournon. V. aussi d'autres études sur notre auteur, études qui, toutefois, ne s'occupent pas en particulier des romans et du thème que nous analysons ici: G. Polizzi, Le Moyen de (ne pas) parvenir: Béroalde de Verville auteur mineur?, «Littératures classiques» 31, automne 1997, pp. 27-38; E. BuTTERWORTH, Subject to Dispute - Construction of the Author In François Béroalde de Verville's Palais des curieux (1612), «French Forum» 32, Number 3, Fall 2007, pp. 23-37.

(2) Nous nous permettons de renvoyer à notre article: «Les Amours d' Esionne» de Béroalde de Verville: roman-piège, roman-labyrinthe, in Sans autre guide. Mélanges de littérature française de la Renaissance offerts à Marcel Tetel, Paris, Klincksieck, 1999, pp. 255-267. Soulignons aussi que l'analyse du thème céleste 
Pucelle d'Orléans. Nous allons considérer, entre autres, les entités et les forces tant naturelles que surnaturelles qui se trouvent ou se manifestent dans ces espaces, les liens existant entre ces derniers et les hommes, et les éléments ou les épisodes de caractère merveilleux liés à la dimension aérienne que le narrateur introduit dans ses deux ouvrages, dans le but d'étonner et de 'divertir' ses lecteurs et ses lectrices. Il est important de faire cette distinction, puisque souvent les Dames représentent le public principal visé par notre auteur, ainsi que les dédicataires explicitement citées de ses romans ${ }^{3}$.

Nous suivrons un critère chronologique en examinant tout d'abord Les Amours d'Esionne. Il s'agit d'un roman assez particulier puisqu'il constitue une masse narrative unique sans aucune division en chapitres ou en paragraphes, ce qui rend la lecture complexe, voire labyrinthique. Toutefois, s'en dégagent deux thèmes principaux: l'amour et la guerre, ce qui est explicité d'ailleurs par les deux titres de l'œuvre qui parut la même année, en 1597, à Paris, chez Matthieu Guillemot sous le titre que nous avons cité plus haut, et à Tours chez Sebastien Molin avec un frontispice différent: Les Amours d'Æsionne ou le Restablissement de Troye ${ }^{4}$. Cet exemplaire tourangeau met donc en évidence dès la page de titre les deux centres d'intérêt du roman: l'aspect sentimental, qui s'adresse surtout aux Dames, et l'aspect relatif aux luttes armées censé attirer les Chevaliers. Le sous-titre souligne également ces deux thématiques, qu'il entremêle jusqu'à les confondre toutefois: «Où se voyent les hazards des armes, les jalousies, desespoirs, esperances, changemens et passions, que les succez balancent par la vertu». Ce mélange entre amour et guerre est du reste justifié par le fait que très souvent on utilise les mêmes termes pour les deux champs sémantiques: «les hazards des armes» renvoient en effet à la guerre et «jalousies» à l'amour, tandis que «desepoirs, esperances changemens et passions» peuvent relever indifféremment des deux. En ce qui concerne plus particulièrement le thème 'céleste' que nous nous proposons de traiter dans cet article, soulignons que les substantifs «hazards», «changemens» et «succez» renvoient au destin, à la fortune dans son sens latin, c'est-à-dire à quelque chose qui vient d'en haut et qui tombe sur les hommes presque toujours comme foudre. Les thèmes du hasard et de la destinée, comme nous le verrons, sont très présents dans ce roman et remplacent souvent, à la différence de ce que nous remarquerons dans La Pucelle d'Orléans, les interventions explicitement définies comme divines. Au tout début du roman, d'ailleurs, le narrateur lui-même nous présente son roman-fleuve en justifiant son choix de raconter sans interruptions et affirme: «Suyvons les hazards comme ils s'offriront [...] au milieu des alarmes [...]: et retrouvant les larmes, les plaintes, les dedains, les douceurs, les accidens,

nous intéresse particulièrement, puisqu'elle complète et fait pour ainsi dire pendant à une autre étude que nous avons consacrée aux grottes dans La Pucelle d'Orléans et dans Le Voyage des Princes Fortunez, puisque les espaces clos et souterrains ont une importance considérable, eux-aussi, dans la production romanesque de notre auteur. L'article, Il tema della grotta in due romanzi di Béroalde de Verville est publié dans le volume Par les siècles et par les genres-Mélanges en l'bonneur de Giorgetto Giorgi, Paris, Classiques Garnier, 2014, pp. 435-455.

(3) À ce propos, citons, par exemple, la dédicace de La Pucelle d'Orléans à «très vertueuse dame, Madame la mareschalle de La Chastre» (p. 73). Au début du roman, Béroalde affirme, dans le Discours Premier, «C'est icy que les dames y prenant garde sentiront en leurs cœurs les effaits des passions veritables et les chevaliers auront le courage attaint, sçachant les avantures, la prudence, et la valleur de cette magnanime guerriere». On retrouve aussi, au début de ce Discours, une invocation explicite du narrateur à la «Belle qui est l'Astre de [ses] intentions» (p. 81). En ce qui concerne La Pucelle d'Orléans, précisons que toutes les citations et les indications des pages se réfèrent à l'édition de C.F. Wilson et de C.H. Winn, Paris, Champion, 2008.

(4) C'est en effet le titre Le Restablissement de Troye qui revient dans les titres courants de l'édition parisienne de Matthieu Guillemot que nous avons pu lire et dont nous tirons toutes les citations. 
les passions d'Amour, arrestons y selon que le temps, les fortunes, les desseins, les fautes, les discours et les evenements nous le proposeront» (p. 4). La structure de ce roman où alternent la guerre et l'amour est donc elle-même dominée par les hasards et les fortunes. Le narrateur, en évoquant les guerres civiles qui tourmentent son pays à l'époque de la parution de ses romans, fait allusion à la figure d'un berger sous la menace d'un orage: «Nous avons quelquefois veu le triste berger souspirer, et invoquant le ciel ne sçavoir plus où courir [...] et presque desesperé emplir le monde de ses lamentations, sentant l'air tout couvert assembler les pierres du ciel [...], les foudres, les tempestes, les esclairs, le danger courant par le vaste de cette estenduë estre prest à tout perdre que soudain le soleil ouvrant ses yeux à travers l'orage en a destourné l'effort prochain» (pp. 5-6). Dans ce passage du texte, qui a évidemment le but d'offrir au lecteur l'espoir d'une amélioration de la condition de la France, le ciel est tout d'abord évoqué et invoqué en tant que lieu de la divinité et tout de suite après en tant que lieu 'météorologique'. Dans les deux cas, toutefois, il faut souligner que le terme «ciel» est écrit avec l'initiale en minuscule, à la différence de ce que nous remarquerons au cours de l'analyse de La Pucelle d'Orléans où ce mot est presque toujours écrit avec une majuscule, étant donné le lien étroit entre l'héroïne et la dimension chrétienne. Dans Les Amours d' Æsionne une dimension 'métaphysique' n'est d'ailleurs pas absente, mais elle apparaît sous plusieurs formes, dans l'ensemble du roman, où figurent aussi des invocations directes à la divinité, qui sont en réalité des exclamations, comme, par exemple, l'expression «Dieu!» figurant à la page 15 ou à la page 106. Ailleurs, c'est encore le hasard qui domine dans les événements de ce roman, telle que la «Fortune envieuse du bien parfait des humains» (p. 19) qui s'amuse à détruire le bonheur des amants, ou «la promesse de [la] future fortune» (p. 26) d'un des protagonistes, le prince Sigismond, qui, allant «à l'avanture» (p. 27) oublie ses devoirs envers Æsionne, son amoureuse. Le narrateur, en outre, réutilise encore une fois une image proche de celle du berger citée plus haut, qui montre le retour de la lumière après une menace - tout à fait métaphorique - de mauvais temps. Il y parle à la première personne:

Voyageant autresfois après les curiositez qui m'ont transporté, ne donnant repos à mes fantaisies [...], j'ay veu le ciel espoissi, les nuées ramassées approcher presque jusque sur le sourcil le corps du Soleil tout pasle et comme voulant choir sans lumiere, que tout à l'instant levant la veuë il passoit un des enfans du Zephir qui dilattant l'air rendoit le flambeau du Ciel en sa place aussi lumineux qu'en sa plus belle gloire et ainsi rejouit la terre d'une lumiere seraine (pp. 45-46).

Dans ce passage, le ciel est nommé deux fois, la première avec une initiale minuscule et la seconde avec une initiale majuscule, mais la différence n'est pas significative à notre avis, puisque dans les deux cas le narrateur se réfère à sa propre activité de 'curieux' qui recherche sans cesse le savoir et l'inspiration et qui, malgré les difficultés rencontrées sur son parcours, atteint la satisfaction dans l'expression de ses fantaisies grâce au plaisir de l'écriture. Il faut souligner, en tout cas, que le terme 'ciel' figure plusieurs fois dans ce roman, comme, par exemple, dans un passage se référant à la justice, que l'on doit toujours respecter, sans quoi «le ciel fait pleuvoir toutes maledictions» (pp. 261-262) sur un peuple, ou, encore, quand le narrateur affirme «le ciel qui voit les cœurs» des hommes connaît la vérité (p. 271).

En outre, l'héroïne éponyme, Æsionne, fait allusion aux «puissances celestes [qui] sont étranges» (p. 55) et qui, dans le cas présent, président à l'amour des êtres humains. C'est l'une des expressions se référant à des êtres supérieurs, d'ailleurs non connotées dans un sens religieux précis, que nous retrouverons, avec des dénominations variables, dans le cours de ce roman et, en général, dans presque toute la pro- 
duction narrative vervillienne. C'est encore Æsionne elle-même qui, s'adressant à un chevalier, Saramand, qui est tombé amoureux d'elle sans être aimé de retour, affirme: «Si je fay mine de ne croire cela que me dites, vous remplirez le ciel de sermens jurez estrangement, et l'air espoissi de vos plaintes vrayes ou fausses comblera tout de voix regrettantes» (p. 56). Dans ces deux passages du texte, le ciel devient donc un lieu presque païen, siège d'une ou plusieurs entités divines qui dominent les cœurs des hommes et en reçoivent les plaintes amoureuses. Toujours dans le domaine amoureux se situe une autre affirmation d'Æsionne, qui en parlant d'un homme qu'elle a aimé et qui a été «miserablement massacré» par le «desloyal» prince français Sigismond, souligne que ce dernier n'a pas eu crainte de «la vengeance celeste» (p. 67). L'élément des puissances qui président à l'amour revient encore dans la bouche de la princesse Theophante qui cherche à tromper sa rivale en amour, Basilée, et qui s'adresse à cette dernière avec ces mots: «Sage fille de Roy (luy dit-elle) qui sçavez que les intentions de nos cœurs sont guidées par certaines forces superieures, je suis en une angoisse merveilleuse» (pp. 111-112). Tous ces passages mettent pour ainsi dire en scène des forces qui dominent les sentiments des êtres humains, sur lesquelles Béroalde de Verville ne nous fournit pas d'autres renseignements ou éclaircissements, mais dont il est évident qu'elles ne se réfèrent pas directement au Dieu chrétien, à cause de leur forme au pluriel. Si toutefois nous considérons un discours que Sigismond (lequel au cours du roman, tout comme plusieurs personnages béroaldiens, change de nom et de rôle afin de conquérir Basilée) adresse à cette princesse: «face le ciel ce qu'il voudra, rien ne me destournera de mon fidele espoir [...]. Les destinées qui vous ont establie royne de mes desirs, sont la mesme puissance qui m'induit à estre vostre [...] . Croyez que c'est l'Ange de mes affections qui m'addonne à cette rencontre» (p. 122), nous pouvons remarquer que l'image de l'Ange (avec une majuscule) évoque un personnage qui appartient généralement au domaine du merveilleux chrétien. Ce type de créature céleste réapparaîtra aussi plus loin dans ce roman, en tant que «pitoyable Ange de la vie» (p. 201) qui a apporté du ciel un remède contre un poison, ou encore désigné comme «l'Ange de la verité» (p. 208) qui protège un personnage secondaire.

Comme c'est le cas, souvent, dans Les Amours d' Esionne, le narrateur passe plusieurs fois des récits concernant l'amour à ceux qui racontent les exploits guerriers. Dans ces derniers passages l'évocation de l'espace céleste et de la fortune est aussi fréquent; citons quelques exemples: pendant une bataille furieuse «[le] bruit [des armes] fait retentir le ciel et la Mer» (p. 84), «il ne se trouve icy autre consideration que de vaincre sans s'aviser des accidens que la fortune distribue sur les combatans, qui sont pesle mesle enlacez par le fer de leur indignation qui est conduit selon la temerité du hazardeux destin de la guerre executant les determinations du ciel sur les hommes sanguinaires» (p. 87). Le caractère de ce roman mêlé de guerre et d'amour est confirmé, d'ailleurs, par une série de phrases qui le définissent de manière efficace et qui se terminent avec une évocation du 'ciel païen': «C'est icy un vrai Theatre de guerre et d'Amour [...]. Et si nous tournons un peu les yeux par-dessus ceux qui se font double guerre, nous trouverons l'Amour [...]. Ceux qui font la guerre ne se peuvent priver d'Amour, il faut qu'une fureur soit relevée par l'autre [...], aussi Mars sans Venus est comme un Soleil sans lumière» (pp. 159-160).

Il est aussi intéressant de citer quelques morceaux de ce roman qui contiennent des images typiques de l'époque baroque, puisqu'elles renvoient à l'inconstance des esprits volages ou au sentiment d'incertitude vécu parfois par les personnages amoureux et qui se réfèrent directement ou de manière métaphorique à l'élément de l'air. Le premier passage concerne la princesse Basilée, qui «toute tremblante des hazards de son amour, ne sçait qu'estimer de cet esclair qui vivement jettant son estincelle en son ame, ne luy a pas donné loisir de le penser seulement [...] et mesle son amour d'une incertitude de conjectures volages, tant mal fondées que son esprit esperdu va 
voltigeant après des Idées informes, sans se pouvoir resoudre» (p. 127). Ce passage est suivi d'une phrase exprimée par le narrateur lui-même tout de suite après la description que nous venons de citer de l'état d'âme de cette princesse, ce qui confirme, encore un fois, le caractère pour ainsi dire 'ondoyant' et labyrinthique de la structure de ce roman; il affirme, en effet: «nous laisserons voguer Mondorant» autre nom assumé par Sigismond pour cacher son identité pour se joindre à son armée «et nous irons sur les airs du beau transport de nos desirs traversant en un instant où nous trouverons le Prince Lorrain» (p. 128). Enfin, le prince lui-même, très inconstant en amour, est défini par une image typiquement baroque: «il est ainsi que ces venteuses boullettes bricolantes par l'air, qui suivent à bonds violents les rencontres quelles sont, et si d'advanture elles se viennent à joindre à quelque chose solide elles s'evanouissent, et cet air enflé qui faisoit tant de parade se reduit en une petite gouttelette mesprisée» ${ }^{5}$ (p. 150).

Un personnage qui a un rôle très important dans Les Amours d'Æsionne et qui mérite une attention particulière puisqu'il est directement lié au ciel et aux forces qui l'habitent est Flambor, un ermite ${ }^{6}$ à qui le narrateur consacre plusieurs pages dès son apparition au cours de l'intrigue. Il est tout d'abord défini comme «ce vieil repertoire de toutes sciences, ce docte Flambor Prince de doctrine entre les plus experts, admirable artisan à contrefaire ce que sçait nature» (p. 130) et encore comme quelqu'un «[qui a l'] admirable vertu de la toute puissance de celuy qui ne depend que de soy» (p. 132). Flambor est donc comparé à un être très semblable à une divinité, comme il est confirmé aussi par ses autres qualités: «[c'est] un personnage dont l'esprit genereux s'esgaye après des sujets de telle consequence que les plus grands feroient leur fortune par iceux [...]; [c'est] un notable secretaire de nature, dont l'esprit passant les volages idées des conceptions de ces doctes apparens, va glissant dessus et dedans les formes plus accomplies» (p. 134). L'intérieur de sa grotte - habitation habituelle des ermites - reproduit un petit monde admirable, si bien que le narrateur, en s'adressant à ceux qui recherchent un savoir supérieur, affirme: «Âmes cupides si vous aviez gousté la moindre douceur des curiositez que l'air de cette grotte presente, vous oubliriés vous mesmes [...] pour engloutir à longs traits d'œil les delices de ce tabernacle plain de toutes les commoditez de plaisirs» (p. 132), tandis que, pour ce qui concerne l'extérieur, «les arbres d'autour croissans de tous aages sont les cabinets des chantres de l'air [...] et les Zephirs s'entreployans és rameaux delicats des cimes ondoyantes bourdonnent» (p. 133). L'ermite est donc aussi lié aux vents: il est l'un de ces «chantre de l'air» cités par le narrateur.

Flambor est étroitement lié aussi au monde du merveilleux et de l'alchimie, comme d'ailleurs nous le suggère son nom formé par l'union entre 'flamme' ou le verbe 'flamber' avec le terme 'or', mots qui renvoient au feu, l'élément nécessaire pour accomplir le Grand Euvre, et à son but. L'ermite en effet est un «sage qui cognoist tous les replis de la cabale, sçait tous les envelopements de Magie [...], il reüssira des merveilles estranges» ${ }^{7}$ (p. 138), mais surtout c'est un personnage, nous

(5) À propos des images baroques, nous renvoyons à l'une des études fondamentales de J. RoussET, $L a$ Littérature de l'âge baroque en France: Circé et le Paon, Paris, José Corti, 1953. En ce qui concerne particulièrement «le venteuses boullettes» dont parle Béroalde, v. l'article de J.-P. LEROY, L' "Ampoule venteuse", ou de quelques images baroques dans le théâtre d'Antoine de Montchrestien, «Revue d'Histoire Littéraire de la France» 4, 1964, pp. 645-651, très utile même s'il est consacré à un autre écrivain de l'époque baroque.

(6) Nous renvoyons à notre étude: L'ermite détenteur du savoir dans l'œuvre romanesque de Béroalde de Verville, in Littérature et prophéties, «Babel, Revue de littérature française, générale et comparée» 4, 2000, pp. 37-52.

(7) Sur ce thème, v. G. PoLIzzi, Fantômes et contrefaçons dans l'œuvre de Béroalde de Verville: ouvrages virtuels, fictifs et fictionnels, «Renaissance and Reformation / Renaissance et Réforme» 3, vol. 34, Été 2011, pp. 91-110. 
dit le narrateur, qui «[...] avoit communication avec les puissances superieures, et pouvoit non seulement secourir les affligez, aider aux amans, nuire à ses ennemis, ains guider les volontez contraires à son plaisir [...]; il estoit maistre en toutes sciences esquelles il sembloit que mesme les astres le redoutassent, tout luy obeissoit» (p. 180). Il est donc tout à fait lié au ciel et détenteur d'un savoir qui aspire à l'absolu. C'est luimême, d'ailleurs qui l'affirme, en parlant avec un autre personnage: «je vous promets de vous faire voir la puissance que le Ciel m'a communiquée» (p. 185).

Cependant, Flambor, «oracle de parfaites propheties» (p. 352), n'est pas une figure tout à fait positive, dans le sens que d'une part il utilise son savoir presque divin parfois aussi en faveur des ennemis ${ }^{8}$, et que, de l'autre, il semble oublier ses dons particuliers, comme cela est souligné par le narrateur lui-même dans le passage suivant où il se réfère à un personnage qui n'a pas été prévenu d'un péril par le vieil ermite: «Mais j’apperçoy quelque ame mignarde qui veut sçavoir pourquoy Flambor ne l'en advertit, il faut patienter et croire que les intelligences superieures ne donnent pas toujours avertissement si on ne les requiert» (p. 360).

L'ermite est toutefois l'auteur de l'enchantement le plus 'merveilleux' figurant dans ce roman, et pour l'accomplir il se révèle même physiquement très proche du ciel et des esprits qui l'habitent, puisqu'il réussit à s'envoler. Il veut en effet éviter que le duel entre deux des protagonistes, Sigismond et Aronde, ait une fin tragique et surtout réaliser un enchantement qu'il avait promis d'opérer en faveur de la princesse Theofante:

Le prevoyant Flambor qui machine ce qu'il a deliberé avec Theofante, estant sur le terme de faire reussir son charme sçait ce combat qui peut ruiner toutes ses entreprises, parquoy aussi tost il s'avise d'un expedient, les esprits apprestez pour sa deliberation luy servent à cette urgente occasion. Il ne faut pas qu'il perde de temps [...]. Il convient à cet extremité que son art lui face service, il est necessité d'un coup de maistre, il se depesche et ayant apresté une ramasse se met dessus et se fait trainer par les rochers et montagnes de nuées qui flottent sur les airs moyens où elles s'espoissent, se faisant ainsi emporter il monte les traits de son visage et le reste de sa figure à la semblance de la belle Flambiose gloire des Samaritaines, avec luy est une similitude de coche de ville [...] où sied un esprit representant Æsionne soit d'habillement soit de façons et de parole, avec luy est un autre lequel se faisant voir paroist la belle Cypriotte [Basilée] (pp. 391-392).

En montrant aux deux chevaliers en lutte ces images féminines et en particulier celle de Flambiose - une amazone qui sera rappelée aussi dans La Pucelle d'Orléans Flambor réussira en effet à les séparer et à éviter qu'ils s'entretuent, au grand étonnement des présents qui ne voient pas les feintes images féminines créées par l'ermite?.

Une fois accompli cet enchantement, Flambor «donne carriere à ses coursiers venteux portez d'ailes nuageres qui le transportent où il veut laissant le reste du sort aux esprits qui demeurent» (p. 396). Enfin, Flambor est aussi défini comme celui «qui

(8) Flambor, en effet, aide en particulier Théofante, une anti-héroïne cruelle et vindicative.

(9) «Ils se faisoient une violence estreme que voicy une apparance [chacun d'eux ne voyant que celle qui luy estoit ordonnée] qui se monstrant au Prince François en la façon de la vaillante Samaritaine luy presente Basilée le priant de laisser ce duel, elle se met entre les combatants et paroist à Aronde estre Æsionne laquelle il craint d'offencer [...]; Sigismond ayant en respect merveilleux ce qu'il ayme craint de l'outrager $[\ldots]$, ces feintes les destournent, par la reverence qu'ils ont à ce qui leur est present $[\ldots]$ mesmement estimant que ce sont celles qui ont toute puissance sur eux, parquoy en mesme instant, d'une mesme volonté, d'une façon egale et comme s'ils l'eussent projetté, se retirent. Les spectateurs qui ne les ont veus autrement qu'en combatans sont éstonnez de si soudains changement [...], car ils n'ont rien veu de l'artifice de Flambor» (pp. 394-395). 
sçait beaucoup des choses passées et [...] est creu comme un parfaict oracle de bon avis» (pp. 448-449).

Le vieil ermite a pour ainsi dire une homologue féminine beaucoup moins puissante et présente que lui dans le roman, mais qui a toutefois un rapport tout aussi étroit avec le ciel et la divinité. Il s'agit d'une «ancienne Dame» (p. 438) de Samarie: «c'estoit la prudente Melisarde, fine sur toutes les femmes accortes entre celles qui en sçavent, et multipliante en inventions: Aussi pour son sçavoir et experiences elle estoit la troisième de toutes celles du Levant en l'ordre Hierarchique que s'attribuent les Kabalistes qui font estat de pouvoir par charmes et Herbes» (pp. 438-439). Flambiose, la belle et vaillante amazone, qui est tombée amoureuse de Saramand/Aronde après l'avoir refusé, vient chez elle lui demander son aide pour apaiser la peine de son cœur et l'appelle «Sage gouvernante des puissances superieures» (p. 440). Melisarde conseille à Flambiose de monter sur le «grand Olympe» (p. 443) pour chercher une plante sacrée au Soleil qui ne porte:

qu'une fleur de couleur d'or tout au tour, et au milieu il semble qu'elle soit comme estin fondu avec la marcassite Mercuriale [...]; ce milieu est licé en perfection et est le miroir de fidelité [...], si une vierge cueille cette plante durant que la Lune est au signe des Gemeaux, elle se pourra vanter d'avoir un bien inestimable. Vous irez en cet endroit et choisirez cette divine plante qui vous mettra hors de toute peine, car l'ayant devant vous, elle vous representera au vif ce que vous desirez comme vostre intellect le produira en soy mesme.

Quand elle verra Saramand, Flambiose devra lui offrir un bouquet avec un brin de cette fleur lié avec ses propres cheveux et, dès ce moment il sera sous le charme. Dans ce cas aussi nous trouvons une référence très claire à la Kabale et à l'alchimie, grâce aux références à la Lune et à Mercure. Comme nous le verrons aussi dans La Pucelle d'Orléans et dans Le Voyage des Princes Fortunez, ces deux savoirs sont étroitement liés aux 'puissances supérieures' et à leur savoir secret.

À propos du ciel vu dans un sens métaphysique, il faut aussi remarquer que, plus loin dans le roman, la divinité est évoquée et invoquée par le narrateur lui-même, qui est en train de raconter l'amour entre Saramand/Arronde et Flambiose et qui déclare la soumission des hommes et en particulier la sienne propre en tant qu'écrivain:

Il est vray que tousjours nous ne sommes pas maistres de nos deliberations, et qui est le bel esprit qui voudroit croire qu'il n'y eut icy quelque chose de divin? Veu que nous ne pensions au commencement que cette avanture survint, nous y sommes entrez sans dessein particulier, et les destinées nous conduisent en des sentiers qui ne sont cognus qu'à l'auteur de Tout, ô Puissance infinie qui nous permet de nous recreer en ces belles vanitez. Si entrans en cette course nous ne t'avons recherché pour implorer ton aide n'en aiguise pourtant sur nous ton indignation $[\ldots]$, ains nous eslevant à toy veuille que ce monde et ses apparences nous soyent comme ces jeux [...]. Tu as recouvert les serieux conseils de ta gloire d'infinies apparences plus joyeuses que nostre esprit ne se peut imaginer de plaisir (pp. 434-435).

Dans ce passage le narrateur reprend, en la précisant et en l'approfondissant dans un sens plus spécifiquement divin et surnaturel, la déclaration que nous avions citée au début de notre étude à propos du hasard qui conduit Verville dans son entreprise d'écriture. Dans le morceau textuel ci-dessus, il cite encore «les destinées», mais il les rapproche de la volonté du Tout-Puissant, ce qui semble montrer, en s'approchant de sa fin, une orientation plus évidente du travail du narrateur vers une union des forces du hasard avec celles du domaine de la divinité.

Le dénouement en effet confirme cette hypothèse; Les Amours d'ÆEsionne se termine par un épisode de guerre: «Les destinées despecheoient les affaires pour au moyen de nouveau accidens construire divers evenemens, et comme les forces supe- 
rieures agissent l'une vers l'autre balançant peu à peu ce qui doit avenir, les succez se meslent, tout rentre en rumeur» (p. 468). Le Prince Sigismond semble avoir 'rétabli Troye', mais la montagne se fend et tout éclate. Comme c'est le cas dans la plupart des romans vervilliens, le dénouement, en réalité, n'en est pas un, dans le sens que notre auteur a l'habitude d'interrompre de manière soudaine et imprévue sa narration. Le roman en effet se termine avec une image qui renvoie, bien que de manière assez ambiguë, au domaine de l'amour, et qui balance donc l'épisode final de guerre: Flambiose voit un chevalier tombé pendant la bataille et lui soulève «l'acoustrement de teste pour luy donner air, [mais] elle se couvre d'un voile de tristesse tant douloureux que son ame desolée la laisse presque en l'estat de celuy qui est miserablement trebuché près d'elle» (p. 475). Le chevalier est peut-être Saramand? Il est mort ou blessé? Nous n'en saurons jamais rien. En effet, comme c'est le cas dans plusieurs romans béroaldiens, la narration reste inachevée. Apparemment, c'est comme si le sort (ou le ciel?) empêchait en effet l'écrivain d'accomplir son ouvrage ${ }^{10}$.

Le deuxième roman que nous allons examiner est La Pucelle d'Orléans publié en même temps à Paris chez Matthieu Guillemot et à Tours par Sébastien Molin en 1599 ${ }^{11}$. Il faut tout de suite souligner que, à la différence des Amours d'Æsionne, ce texte est divisé en trente-cinq «Discours» chacun précédé de quelques lignes qui en résument le sujet. Ce choix de l'auteur est très positif, puisqu'il facilite du moins en partie la lecture, même si la structure de l'ouvrage reste cependant assez complexe. Comme nous l'avons déjà annoncé précédemment, dans cet ouvrage les rapports des personnages et du narrateur lui-même avec la fortune, le Ciel et la divinité sont beaucoup plus connotés dans un sens religieux, étant donné le sujet que Béroalde y traite. En effet cette remarque pourrait apparaître superflue, puisque la Pucelle est une sainte, mais il faut préciser que bien que l'héroïne éponyme - comme nous l'avons plusieurs fois remarqué dans les études que nous avons consacrées à ce roman ${ }^{12}$ soit inspirée de très près de Jeanne d'Arc et des événements de sa vie, notre auteur introduit dans l'intrigue plusieurs épisodes imaginaires concernant la protagoniste, mais aussi de nombreux personnages et histoires secondaires, ce qui fait de cet ouvrage, comme dans le cas des Amours d' $E$ sionne, un mélange d'histoires d'amour et de guerre, toujours dans le but de 'divertir' ses lecteurs: les Dames et les Chevaliers. Ce roman, d'ailleurs, est un hybride sous plusieurs points de vue: non seulement Béroalde traite d'amour et de guerre, mais aussi, comme nous avons déjà rappelé plus haut, d'Histoire et de fiction, de religion et de merveilleux. Et c'est vraiment ce 'syncrétisme' entre sacré et profane, entre Ciel et fortune qui caractérise en particulier La Pucelle d'Orléans. En outre, dès le frontispice, nous lisons: «Sous le sujet de cette magnanime Pucelle est représentée une fille vaillante, sçavante et belle» et nous sommes informés que la protagoniste cache pour ainsi dire une autre femme, celle dont l'auteur est amoureux et à qui il s'adresse en l'appelant au tout début du texte «l'Astre de mes intentions» (p. 81) et «l'Astre de ma vie» (p. 82) et en établissant donc tout de suite un lien entre la femme et le ciel. Il s'adresse ensuite aux Dames

(10) Dans L'Histoire veritable ou Le Voyage des Princes Fortunez, par exemple, ce sera la douleur à cause de la mort soudaine et violente d'Henri IV qui empêchera le narrateur d'accomplir son travail, ou du moins c'est ce qu'il déclare.

(11) Il s'agit de la même impression parue avec deux pages de titre différentes.

(12) Nous renvoyons, entre autres, à deux de nos études consacrées à ce roman: «La Pucelle d'Orléans» di Béroalde de Verville: tra storia e romanzo, in Scritture dell'impegno dal Rinascimento all'età barocca, Fasano, Schena editore, 1997, pp. 135-146, et «La Pucelle d'Orléans» di Béroalde de Verville o la storia deformata, in Disarmonia bruttezza e bizzarria nel Rinascimento, Florence, Franco Cesati Editore, 1998, pp. 199-213. V. aussi F. GreIner, "La Pucelle d'Orléans" de Béroalde de Verville et l'idéal de la femme forte, in Images de Jeanne d'Arc, Paris, Puf, 2000, pp. 133-142. 
d'Orléans, à qui il a dédié son roman, en affirmant: «Voyez renaistre ce miracle de son age» (ibid.) et il invoque les belles «Ames» (p. 83) de ses destinataires pour qu'elles fassent «profit manifeste du bien qui [leur] est offert, et duquel, dit-il, j'espere tel loyer que le Ciel ne me frustra point de mes esperances» (ibid.). Dans la presque totalité des occurrences de ce terme, dans La Pucelle d'Orléans, le Ciel sera écrit avec une initiale majuscule, ce qui connote ce mot dans un sens décidément religieux. Toutefois, même dans ce texte, tout comme il le fait souvent dans d'autres romans, le narrateur souligne l'importance des destinées qui influencent avec force et même changent l'orientation de sa narration: «Mais [...] les destinées se preparent autrement que nos desseins ne les pensent suyvre, et [...] souvent nos propositions sont destournées au moment que noz conceptions s'y attendent le moins [...]» (p. 85). Il est donc possible de constater que cette idée de la narration qui est dirigée de l'extérieur et d'en haut sans que l'écrivain puisse réagir figure très souvent dans la production béroaldienne. Rappelons aussi que, dans la même page, un chevalier se plaint de «sa miserable fortune» et s'en remet à dieu par l'exclamation «Pleust à Dieu», pour qu'il soit épargné du déshonneur.

La protagoniste, qui assume plusieurs identités et plusieurs noms au cours de la narration est aussi comparée, au cours d'un tournoi auquel elle participe déguisée en chevalier, à «une nouvelle Minerve» (p. 89) et, deux pages après, à «un si beau Soleil», ce qui met en évidence à la fois sa ressemblance avec la déesse armée et son lien étroit avec le Ciel. En effet, même si la Pucelle est vue aussi comme une femme qui suscite l'amour dans le cœur de plusieurs protagonistes, elle reste toujours chaste et n'est jamais touchée à son tour par le sentiment amoureux. Elle est protégée par son «Bon Ange» et «ne descherra jamais de son honneur, seul Soleil de son ame» (p. 97). Les anges sont donc présents aussi dans cet ouvrage et l'on verra que l'un d'eux sera aussi le protagoniste d'un épisode féérique. Comme c'est le cas dans Les Amours d' Esionne, dans La Pucelle d'Orléans aussi nous retrouvons des expressions qui se réfèrent à des entités divines sans que ces dernières soient jamais définies de manière plus approfondie. Le Discours III, par exemple, raconte, entre autres, un songe de la Pucelle qui est très intéressant en ce qui concerne le sujet que nous avons décidé de traiter. Le narrateur affirme que «les intelligences superieures» (p. 98) qui conspiraient au bien de la France ont fait endormir la jeune fille qui «[entre] en un songe notable» (p. 99) et il se pose une question: «Et qui sçait si nos esprits communiquans aux puissances separées apprennent des secrets en repos qui ne se peuvent comprendre parmy les troubles du jour?». Il s'agit d'un songe étroitement lié au Ciel et qui est aussi prémonitoire, puisqu'il annonce sous un sens métaphorique une aventure de la protagoniste et le rôle qu'elle jouera dans l'Histoire de France. En effet, l'âme de l'héroïne «se guindant avec ses pensées et se levant sur le plus haut de l'air, luy fut advis qu'estant eslevée sur les airs dans sa galere celeste elle voyoit sa mere, dans une autre faisant diverses passades par le vaste mihumide des nuées et qu'en ses diverses bricolles ondoyantes en l'air elle estoit tresbuchée au milieu de la France ou elle estoit en grande peine implorant le secours du Ciel» (pp. 99-100). L'image de sa mère lui apparaît et lui demande de la défendre contre les anglais, mais la Pucelle ne sait pas comment lui obéir, vu qu'elle est sans armes, et se désespère, mais «Comme cet accident la traversoit voicy la semblance d'un ange armé [...] qui la prenant par la main luy dict qu'elle le desarmat et endossat son harnois, ce qu'elle fit, adoncque ainsi que forcenée pour la violence que l'on commettoit vers sa mere se jetta dans l'armée ennemie» (p. 100). À son réveil la jeune fille «se mit à s'arraisonner soy-mesme sur cette forme de vision à quoy souvent elle pensa et suyvant ce qu'elle deliberoit recogneut que le Ciel l'admonestoit que sa mere cheute en France luy signifioit que c'estoit son affection qui devoit y tendre, comme à sa mere naturelle, et pourtant que ses destinées l'appelloyent pour y venir servir le roy» (ibid.). C'est donc le Ciel lui- 
même - bien que l'on ne puisse définir avec précision s'il s'agit du Dieu chrétien - qui inspire à la Pucelle sa mission et, en même temps, l'aide à comprendre la signification cachée de son rêve. Voilà un exemple significatif qui nous montre de quelle façon Béroalde se sert de l'histoire bien connue de Jeanne d'Arc qui 'entendait les voix', en y ajoutant des éléments romanesques, tel que l'apparition de l'ange armé et le voyage dans la galère céleste que nous retrouverons encore au cours de la narration.

Remarquons en outre que le Roi Charles VII lui-même et sa maîtresse Agnès Sorel évoquent l'un après l'autre le Ciel et les destinées, ce qui montre l'importance de ce thème de l'union des deux forces dans ce roman aussi. Le Roi en effet, répond au chevalier Brochard ${ }^{13}$, qui lui reproche d'être trop paresseux: «J'espère que le Ciel me donnera moyen un jour ayant recueilli mes esprits, ma mauvaise fortune se lassant de me poursuyvre, de vous recongoistre» (p. 109). Agnès Sorel, cherche à son tour de pousser le souverain à agir contre les anglais: «Avez-vous envie de tromper le Ciel, troubler les destinées, et perdre tout mon bon heur?» (pp. 110-111).

La présence du merveilleux lié au ciel et aux planètes se révèle dans ce roman grâce à un objet possédé par la Pucelle qui lui permet de reconnaître le Roi entre plusieurs chevaliers, tout en ne l'ayant jamais vu: il s'agit du «talisman de cognoissance» (p. 113) dont l'inventeur avait appris «la science des Ægyptiens, les preceptes des Kaldeans, et les pratiques des Arabes» (ibid.), qui permettait de voir en songe le présent et le passé. Béroalde nous offre une longue et complexe description du talisman, qu'il définit comme l'un des «artifices transcendans» (p. 115) et qui exerce son pouvoir grâce à la lune, le soleil, Mercure, Saturne et plusieurs constellations, ce qui le relie donc de manière très étroite à notre thème céleste. Toujours dans ce contexte de connaissance magico-religieuse nous pouvons situer la présence des Druides, assez fréquente dans la production narrative vervillienne, dont la doctrine se base sur l'observation des mouvements des astres et qui mettent en communication le Ciel avec les hommes ${ }^{14}$.

L'épisode historique, qui décrit l'interrogatoire de la Pucelle de la part des théologiens avant qu'elle parte pour la guerre contre les Anglais, est riche d'expressions qui nomment Dieu et le Ciel dans un sens religieux chrétien très défini. Nous en citons quelques-unes: «elle cuidoit estre avoüée de DIEU et si elle pouvoit promettre au roy cette delivrance» (p. 134); «Chaqu'un est obligé par le commandement de DIEU d'apporter ce qu'il peut [...]. J'ay dès mon enfance esté addonnée aux armes, j'y ay eu une inclination qui m'est plus venuë du Ciel que de coustume [...]. Je me contente de ce que Dieu m'a donné [...]. Je ne promets rien que ce que je pourray avec l'ayde de Dieu» (pp. 134-135). «Les Orleanois [...] avoient veu en la Pucelle la delivrante main de DIEU» ${ }^{15}$. De plus, l'hérö̈ne elle-même est comparée plusieurs fois «à un beau Soleil parmy les astres» (p. 150) et définie comme «Plus douce que le jour, plus belle que les Cieux» (p. 152), mais, d'autre part, comme «cette chaste Minerve trionfant de l'Amour, des hommes et des dieux» (p. 154), ce qui montre le mélange entre religion chrétienne et monde païen que nous avons déjà remarqué. Cet aspect est important puisqu'il souligne la double nature de la Pucelle, qui est à la fois une femme chaste et inspirée de Dieu mais qui inspire à son tour des amours, bien que toujours respectueux, dans le cœur des hommes. Il faut en effet rappeler, en passant,

(13) Le personnage de ce chevalier est introduit par Verville dans cette œuvre pour rendre hommage à son mécène dont le nom était Pierre Brochard.

(14) Cfr. p. 129.

(15) Soulignons que dans les trois exemples que nous avons cités ci-dessus, et dans les autres qui suivront, le terme DIEU est écrit en lettres capitales dans le texte. 
que l'héroïne cache pour ainsi dire «sous son écorce» - expression qui revient souvent sous la plume de Verville dans ses romans - la Dame aimée par l'auteur.

Après cette partie du roman où sont racontés les premiers exploits de la protagoniste, commence une série de «Discours» où le narrateur invente de toutes pièces la naissance et la jeunesse 'mythique' de Jeanne qui voit le jour dans l'île de Sympsiquée, lieu fantastique défini comme «un sejour [...] celeste» ${ }^{16}$ (p. 178), le «Paradis du monde» (p. 184) et «un lieu de miracles naturels et artificiels» (p. 188) où le noble chevalier français Borandor arrive aventureusement. Il y rencontrera la belle Armeliane, avec laquelle, après plusieurs années d'épreuves de fidélité, il peut se marier. De ces épousailles «est issuë la Pucelle» (p. 183). Une fois devenue jeune fille, la protagoniste devient l'objet d'amour d'Arcosandre, amour au caractère très courtois et même sacré, comme il est démontré par les nombreux vers que le chevalier dédie à la protagoniste, où il déclare plusieurs fois le lien solide entre ses sentiments et la divinité: «Par ton moyen j'ay veu l'air, la terre et les Cieux» (p. 192); «Mon amour tout divin, est par-dessus nature | Vous en estes l'object, le Ciel en est l'auteur. I Vos yeux m'ont allumé de flammes eternelles, I De celestes desirs vous m'avez excité» (pp. 192-194). Cet amour est toutefois destiné à ne pas être satisfait, étant donné l'avenir, déjà écrit par le Ciel, qui attend l'héroïne. C'est l'occasion, pour le narrateur, de se plaindre de son sort de 'mal aimé' grâce à des images qui renvoient au vol et à Cupidon: «J'ay pitié de toy pauvre amant, je m'abuse c'est de moy que je devrois prendre pitié pour me retirer du glissant qui m'emporte. [...] Mais quoy nous vollons par les airs après nostre conducteur æslé, et faisans des passades dans le vuide qui n'a point de solidité nous traçons après l'incertitude!» (pp. 203-204).

La Pucelle est destinée par les lois de l'île de Sympsiquée à accomplir des actes généreux et glorieux dans la terre d'origine de son père et doit donc partir. Ce qui est intéressant pour notre analyse c'est le moyen qu'elle utilise pour effectuer son voyage vers la France, en partant de l'endroit utopique où elle a vu le jour:

Attalonde, grande ayeule d'Armeliane [...] avoit autresfois inventé une galere celeste dont l'industrie estoit non seulement admirable et magnifique, mais d'un usage de grand profit et commodité. Par l'ayde de cette galere on pouvoir s'eslever sur l'espors des aers plus solides au haut ainsi que sur les mers, et maniant un timon qui faisoit mouvoir les organes on se conduisoit à plaisir, et le vaisseau se balanceant en ses proportions suyvoit la route que le vent luy donnoit par l'adresse de la conduite et du mouvement. Armeliane avoit bien conservé ce vaisseau, lequel elle donna à sa fille avec honneste compagnie pour la servir et assister. [...] La plus ancienne des demoyselle estoit Aldonze la Sage, qui avoit apris [...] à guider la galere (pp. 212-213).

Armeliane s'adresse à sa fille en lui disant: «tu dois estre l'CEil et l'Astre plus clair, le Ciel t'envoye où tes destinées t'apellent, sois toujours prudente en tes actions, et craignant le Tout-Puissant, avance-toy à bien faire selon ce qui luy est agreable» (p. 215). Ainsi:

la Pucelle entrée en sa galere se donne route selon que l'intention se preparoit, ores sur le plain des mers et ores par le vague des airs, tant que relevée plus haut sur les Gaules elle esleut lieu propre à sa descente pour s'accoustumer à nouveau pays. [...] Le vaisseau sagement

(16) Rappelons que dans un épisode secondaire qui se déroule sur l'île de Sympsiquée, une «savante magicienne» (p. 176), Marigotte, «fait un charme par le moyen duquel elle assemble [quelques] esprits qui [...] la transportent par l'air à sa volonté, eslevée bien haut» (p. 177). 
conduit vint se rendre dans les Ardennes pres les limites de la Lorraine, en un endroit assez couvert et où il sembloit que la fortune eut preparé le logis de la Pucelle.

Tous ces épisodes relatifs à l'histoire merveilleuse des origines de la protagoniste constituent à l'intérieur de la narration une longue analepse qui enrichit considérablement le côté romanesque attribué par le narrateur à son héroïne et, en même temps, met considérablement en relief le caractère de cette dernière à la fois glorieux et 'céleste', grâce en particulier à son expérience de vol sur la galère - voyage vers le haut qui a une signification évidemment initiatique à la mission qu'elle doit accomplir - et grâce aux liens que tous ses proches évoquent entre elle et la divinité. Cette partie est donc celle où la double nature fictionnelle et historique de cet ouvrage et de sa protagoniste apparaissent de manière plus évidente. En outre, rappelons que dans le songe de la Pucelle dont nous avons traité précédemment, la galère céleste avait déjà fait son apparition. Cette dernière, une fois atterrie dans une forêt, devient «une belle et honneste petite maison [...]» presque «un petit racourcy de palais» (p. 216) où l'héroïne assume l'image de Diane, typique du monde pastoral: «Son habit de chasse la rendoit presque semblable à la déesse des forests que l'on voit courant au travers des boys où ses nimfes l'accompagnent» (ibid.).

L'histoire reprend de manière plus linéaire après cette analepse, et le narrateur nous présente un autre personnage masculin, Filion, qui, en tant qu'«amant désolé mal traicté de sa destinée amoureuse» (p. 226) adresse des prières «aux intelligences superieures $[\ldots]$ se trouvant en oportunité de dilater commodement son ame devant les puissances souveraines» (ibid.). Ces entités non définies reviennent donc souvent dans La Pucelle d'Orléans et se juxtaposent sans problème aux nombreuses évocations de la divinité chrétienne qui ont lieu après quelques pages, où Charles VII, lorsque «la Pucelle paroissoit, creut que DIEU luy avoit par un gracieux miracle envoyé cette magnanime liberatrice» (p. 239). En effet, le Roi, qui s'était vu «si prez d'une extresme ruyne [...] et puis si soudain restably par un manifeste secours du Ciel mis en la main de la Pucelle, se reduit au devoir d'en remercier l'Eternel. [...] Jamais le bien ne nous est donné sans qu'il soit payé pource que le Ciel nous vend tout au pris du travail et des hazards» (p. 248).

Comme nous l'avons annoncé plus haut, le narrateur introduit un autre épisode ayant comme protagoniste un ange. La Pucelle a été blessée à la cuisse pendant une bataille et Colizerpe, l'une des demoiselles qui l'ont accompagnée en France et qui est experte dans la chirurgie et dans les pouvoir des herbes, part pour chercher un remède dans la forêt. Mais «un Ange celeste dolent de voir l'honneur des François en danger d'estre si tost esteint par l'artifice de l'esprit de perdition, sçachant qu'à la blessure de la Pucelle plus faicte par envie que par hazard, il s'esmouvoit une efficace qui n'estoir point naturelle, pour laquelle empescher il voulut y mettre ordre» (p. 249). Cet Ange, qui avait été chargé d'aider l'héroïne française, avait en effet découvert qu'un milord anglais, grand magicien, avait «par les plus rares effets de son art, arresté un esprit malin adversaire de l'Ange françoys» (ibid.) qui s'était transformé en chevalier ennemi et avait blessé la protagoniste avec une épée empoisonnée. L'Ange, alors, fait endormir Colizerpe et en en assumant l'aspect de la jeune fille, prend soin de la Pucelle: «La main angelique appliquoit en apparence les herbes recherchées, mais occultement infusoit en la playe une liqueur heureuse qui garissoit parfaictement» (p. 250). Cet épisode, qui réunit lui aussi le merveilleux chrétien au merveilleux romanesque, se conclut avec une digression du narrateur qui établit une distinction entre les «Anges saints» et les «daimons iniques» (p. 251) ennemis des humains, et affirme: «au contraire de ce qui est de l'Eternel duquel les dons sont gratuits, le mauvais esprit est plain d'artifices cruels [...], mais à ce que baille le bon Ange on y recognoist toute simplicité, aussi jamais il ne pratique d'inventions que la 
fin n'en tende à l'honneur de Dieu» (ibid.). Le même Ange «qui tient l'intelligence du gouvernement de la Pucelle» (p. 282) reviendra dans un autre épisode où il chargera Colizerpe d'aller sauver une dame, «la sotte Stefise» (ibid.) qui avait appris comment conduire la galère céleste, mais qui, une fois entrée dans le vaisseau et montée dans les airs, ne sait plus comment atterrir. Et le narrateur de commenter: «Il y aura quelque ame chatouilleuse qui dira que cet Ange pouvoit bien faire ce qu'il avoit apris à Colizerpe. Ne vous abusez poinct, il n'est pas seant que tels espris derogent à leur grandeur, et que toujours ils facent absolument et immediatement ce qui leur est permis!» (p. 283). Dans La Pucelle d'Orléans, donc, l'Ange est une créature qui, suivant la nature du roman lui-même, se situe à mi-chemin entre le religieux et le magique, en accomplissant en même temps des miracles, des charmes et des feintes.

Ce roman, tout comme Les Amours d'Esionne, se conclut de manière abrupte et inattendue ${ }^{17}$, au moment où le narrateur devrait raconter l'arrestation de la Pucelle de la part des anglais. Malheureusement, en effet, la Belle aimée par notre auteur le quitte et, nous dit-il: «oubliant le contentement qu'elle prenoit à l'ær de mes discours, je ne sais quelle nuée luy a passé devant les yeux qui l'a renduë ingrate à mes humilité, dedaigneuse à mes offertes, et destournée de la félicité qu'elle me presenta recevant mes vœux. [...]. Et pour tesmoigner mes fidelitez et mon ennuy je jette à bas mon courage» (p. 351). Comme c'est le cas dans le dénouement manqué du premier roman que nous avons analysé, même dans La Pucelle d'Orléans nous ne saurons jamais pourquoi la Dame qui se cachait derrière l'héroïne française a renié son amour.

Pour conclure, nous pouvons donc affirmer que les deux ouvrages que nous avons analysés dans le but de mettre en évidence la thématique du ciel, présentent d'une part des caractères communs en ce qui concerne leurs centres d'intérêt, l'amour et la guerre. De l'autre, par ailleurs, ils diffèrent du moins partiellement pour ce qui est justement de l'évocation de l'espace céleste, liée surtout à l'action de la fortune et du destin dans Les Amours d'Esionne, tandis que dans La Pucelle d'Orléans, à cause aussi du sujet en partie lié à l'histoire de Jeanne d'Arc, cette évocation est plus étroitement inspirée par le thème religieux et chrétien. Il n'est peut-être pas inutile de rappeler, à ce propos, que Béroalde de Verville a eu pendant son existence, un rapport assez complexe avec son appartenance religieuse: né protestant, il était ensuite passé au catholicisme en devenant aussi chanoine à l'église de Saint-Gatien de Tours, pour revenir encore, vers la fin de sa vie, au protestantisme. Ce sentiment religieux pour ainsi dire ondoyant ne semble pourtant pas avoir exercé une influence déterminante sur son activité d'écrivain: les Anges, par exemple, figurant dans les deux ouvrages, possèdent des caractéristiques liées en même temps à un aspect sacré et à un aspect 'merveilleux', les envols dans les airs ont presque toujours un caractère féérique plutôt que religieux. Le ciel est donc à la fois le synonyme de Dieu et l'endroit riche de

(17) Toujours à propos du thème céleste, nous tenons à citer un épisode du Voyage des Princes Fortunez (Paris, Pierre Chevalier, 1610) où l'élément de la 'fortune' apparaît aussi dans le titre, mais que nous avons choisi de ne pas analyser de manière plus ample, puisque son caractère surtout alchimique et stéganographique nous aurait éloignés du chemin que nous voulions suivre dans cette étude. Toutefois, cet épisode mérite d'être rappelé parce qu'il traite du rapport entre les hommes et le ciel selon la catégorie du merveilleux, mais d'une manière anticipatrice de la modernité. Un couple d'amants a été séparé, mais réussissent à communiquer et même à se voir, puisqu'«ils avaient apris [...] un merveilleux secret, qui est que par le moyen d'une bague qu'ils avoient chacun la sienne, ils pouvoient la presentant à la lune, voir ce qu'ils desireroient des actions l'un de l'autre. La nuict venue Aderite [...] se mit à la fenestre comme pour prendre un peu d'air, et opposant la bague à la lune clairrayante, la tourna et vira tant qu'elle avisa son desiré Giseol, [... alors elle fit briller la bague sur ses yeux dont ils s'apperceut: parquoy il regarda en la lune qui luy monstra sa maistresse dans le jardin du palais, ce qui le consola infiniment» (p. 666). Dans son caractère merveilleux, cet épisode semble étonnamment anticiper une communication, aujourd'hui possible, grâce à un satellite artificiel lancé dans l'espace. 
secrets auquel sont liés les personnages des sages et des enchanteurs, comme par exemple Flambor. Comme nous avons déjà rappelé, si la grotte, espace chtonien par excellence, est directement associé à l'athanor ou vase alchimique et donc à l'aspiration à atteindre un savoir secret, le ciel et les espaces célestes en général constituent le complément indispensable aux espaces clos et terrestres pour que le désir de connaissance soit, sinon complètement assouvi, du moins recherché avec force en s'élevant vers le haut et le divin.

Dans les deux cas l'instrument pour chercher le savoir est constitué pour Béroalde de Verville par l'activité de l'écriture. Toutefois, comme nous l'avons déjà souligné, il nous avoue que très souvent c'est le hasard qui conduit sa plume, et presque toujours c'est un fait imprévisible qui l'arrête soudainement. Dans les deux romans analysés et dans d'autres aussi, en effet, le narrateur nous laisse pour ainsi dire 'sur notre faim': il semble ne pas pouvoir arriver, ou peut-être ne le veut-il pas, à dénouer les nœuds très complexes de sa trame narrative.

Il faut tout de même préciser que cette attitude à ne pas terminer la narration se retrouve d'ailleurs assez souvent dans les romans de l'époque de Béroalde de Verville, puisque, simplement, le dénouement n'était pas ressenti comme indispensable. Cependant, chez notre auteur, il s'agit d'un choix constant dans toutes ses œuvres de caractère romanesque. Nous pouvons affirmer, en tout cas, que cette posture narrative béroaldienne n'est pas un simple escamotage pour ne pas devoir dénouer tous les fils des intrigues extrêmement compliquées et parfois mystérieuses qui caractérisent ses textes, mais qu'il s'agit d'un choix délibéré. Il suffit de penser à son ouvrage le plus connu et étudié, Le Moyen de Parvenir qui n'a ni début ni fin, pour ainsi dire, puisqu'il détruit toutes les structures traditionnelles du roman: une trame linéaire, des personnages bien définis, une cohérence dans la narration. À notre avis, dans ses textes qui précèdent ce texte, notre auteur annonce déjà ce caractère atypique du Parvenir, notamment dans la fluctuation des caractères et des identités de ses protagonistes et dans la complexité de ses narrations ${ }^{18}$.

Pour ce qui concerne enfin l'élément que nous avons traité dans ce travail, le ciel, il faut aussi souligner que, dès le début de sa carrière de poète et d'écrivain, Verville s'est distingué par un syncrétisme très accentué. Comme nous espérons l'avoir montré, il n'est donc pas possible d'établir une seule nature de la divinité et du hasard, puisque cet auteur mêle toujours, selon ses exigences d'écriture, vision chrétienne et vision païenne, Dieu ou les dieux, volonté divine ou intelligences supérieures, religion et alchimie. Comme il le dit souvent dans ses pièces liminaires ou ses avis au lecteur, son œuvre est ainsi une sorte d'anamorphose, où une écorce superficielle couvre presque toujours un sens caché ${ }^{19}$, que le lecteur doit apprendre à découvrir selon la bonne perspective et l'exact point de vue.

DANIELA MAURI

Università degli Studi di Milano

(18) V. à ce propos, notre article L'écriture alchimique de Béroalde de Verville romancier, in Perspectives de la recherche sur le genre narratif français du dix-septième siècle, Paris-Genève, Edizioni Ets - Editions Slatkine, 2000, pp. 53-77.

(19) Cfr., par exemple, la dédicace à François de Guesle, dans Les Amours d' Esionne, où Béroalde souligne que «la mignarde superfice de cet œuvre» et «cette escorce reparée des traits delicieux que j’ay choisis» couvrent quelque chose de «plus serieux que leur aparence». 